\title{
Chitosan composites nerve conduits
}

\author{
Yifan Chen $^{1, \mathrm{a}}$ \\ ${ }^{1}$ Department of Biomedical engineering, Stevens Institute of Technology, Hoboken, U.S.A.
}

\begin{abstract}
Traumatic nerve defect affected over 200,00 people in the US. As it affected so many people, autografts as the golden standard solution have their limitations. Nerve conduits is a new method and the materials for conduits establishing contain biological materials, synthetic materials and composite materials. Among these categories, composite conduits appear better regeneration ability. Also, chitosan demonstrated have the ability to help nerve regeneration. Thus, chitosan composite may be a good choice in the future. This article compares six kinds of chitosan composite: Laminin-chitosan-PLGA, Chitosan-Zinc Oxide Nanocomposite, PCL/Chitosan/Verapamil, Chitosan/ hyaluronic acid conduit, Chitosan/simvastatin/Pluronic F-127 hydrogel and Chitin/ Carboxymethyl (CM)-chitosan by conduit structure, establishment method, sciatic functional index (SFI), electrophysiology and histology data. This article concludes that chitosan-drug-drug delivery system showed the best recovery result.
\end{abstract}

\section{Introduction}

Traumatic nerve defect has been reported to affect over 200,000 people in the US [1]. The traffic accident is the most common reason causing trauma nerve defect. The patient will suffer a lot like loss of sensory or motor abilities [2]. Therefore, a biomedical scientist should pay great attention to traumatic nerve injuries and nerve regeneration.

Several solutions have been used in recent years. These solutions can be divided into three main categories: grafts, cell culture regeneration methods, and nerve conduits to bridge the nerve gaps. To grafts, an autograft is considered as the "golden standard" for nerve regeneration, while there is no enough nerve tissue for autograft to fit different injuries with different sites and length [1]-[3]. Besides, autograft will cause secondary damage to the patients [4]. In addition to autografts, allograft and Xenograft may cause patients' immune response. Nerve conduits is an innovative solution that comes out in recent years, which can bridge two sides of the nerve gap then help nerve regenerate from the proximal site. Cell culture method often combines with other approaches like grafts and nerve conduits [4]. Schwann cell has been proved to help nerve regrowth. Nerve conduits method is gradually replacing the principal place of autografts and will be widely used in the future.

Nerve conduits' good function, high biocompatibility, controllable degradation rate, non-toxicity, and affordable cost give them advantages when compared with other methods [2], [5], [6]. The conduits can guide axons sprouting from the proximal part, provide a platform or a microenvironment for the diffusion of cytokines and neurotrophic which are secreted by the injured nerve stump, what is more, they can help prevent from infiltration of fibroblast and the formation of fibro tissue. All the materials used for constructing nerve conduits have been proved to be friendly to the human body and have proper degradation rate. The cost for the nerve conduit is affordable to most people. All these characteristics make nerve conduits have advantages over other methods.

As A. Muheremu and Q. Ao [6] mentioned that nerve conduits' qualities could be decided by structure, length and the most important one is the material choice. The material choice can be biological materials, synthetic materials, and hybrids materials [2]. In A. Muheremu and Q. Ao's article [6]: When comparing different materials, the most important physical properties are flexibility, permeability, swelling, and rate of degradation. In addition to physical properties, some in vivo test and histology data like Sciatic Functional Index (SFI), the number of myelinated axons and G-ratio can also be considered to compare which material is optimal [7]-[10]. This article will compare different nerve conduit materials and determine if one material is favorable over another in particular situations.

\section{Nerve conduits materials review}

Recent nerve conduits can be divided into three major categories: biological materials, synthetic materials, and composites.

Biological materials used in tissue engineering could often be protein or polysaccharides with biocompatibility, low-toxicity and hydrophilic surface for cell growth and proliferation. Natural materials have high bioactive properties allowing better interactions between the cells and the conduits. This kind of properties can help enhance the proliferation of the cells and tissue

\footnotetext{
a Corresponding author: ychen168@stevens.edu
} 
regeneration. ECM protein and other types of protein have been used to construct a nerve conduit for three decades. Type 1 collagen is the most popular material used to develop the nerve conduit. NeuroFlex ${ }^{\mathrm{TM}}$ and NeuroMatrix $^{\mathrm{TM}}$ [2] are two examples of collagen nerve conduits which are already approved by the FDA. Both of them are semipermeable, resorbable, flexible tubular collagen matrix. They have macro-pore for nutrient transfer, and semi-permeability prevents fibroblast migration and inflammation formation. Gelatin is denatured collagen, so it is easier to fabricate and preserve it. As an example, when making crosslink between gelatin and genipin, the gelatin-genipin nerve conduit (GGC) performed stability regeneration rate in longer nerve gap regeneration [11]. Other examples like Gelatin-chitosan and gelatin- proanthocyanidin are also proved to have the function of helping nerve regeneration[12]. Additional protein materials like Fibronectin, Silk fibroin, and keratin et al. polysaccharides like chitosan and chitin will be talked in the main body.

Synthetic materials can be divided into biodegradable and nonbiodegradable materials. Biodegradable synthetic materials due to its biocompatibility and do not need a second surgery are widely used. Biodegradable polyesters are very popular in recent years because 1) they will not stimulate immune responses because of their biocompatibility, 2) they often have exceptional mechanical properties and controllable degradation rates with proper construct method and structure design, 3) they are able to enhance the tissue ingrowth, 4) their byproduct of degradation is water, which has no harm to human body. PCL can be dissolved in hexafluoroisopropanol (HFIP), then establish fibers very efficiently with high quality; these fibers can help nerve cell regrowth. As C. Oliveira et al3 showed that PLA could help organize the better distribution of nerves axons. PLGA is a combination of PGA and PLA, the advantage of PLGA is that the rate of degradation can be controlled by changing the ratio of PLA to PGA, while more PLA means higher hydrophobicity, which will make cell challenging to attach and grow on PLGA sheet. Nondegradable synthetic material Polyvinyl alcohol (PVA) is water-soluble with good spin ability. PVA has high mechanical properties and relatively low degradation kinetics[13].

Composite nerve conduit materials have they are advantageous over single biological and synthetic material. The surface characteristic of hybrid material can be easier for cell growth, proliferation and differentiation because of hydrophilic biological materials coated. The main structure can be constructed by synthetic material because of their excellent mechanical properties. According to three main categories of biological materials, hybrid materials can be divided into polyester with synthetic materials, Polysaccharides with synthetic biomaterials and protein with synthetic materials. Chitosan and chitin belong to polysaccharides, and this article tries to focus on chitosan and chitin composites, chitosan and chitin with synthetic materials, materials with cells and materials with drug delivery system, to compare different chitosan and chitin nerve conduit and determine if one material is favorable over another in particular situations. Comparisons are in Table 1.

Chitosan is a kind of polysaccharides biological material; some research has evidenced that chitosan has the ability to help nerve regeneration. Chitosan also has excellent biocompatibility, non-toxicity, accurately rate of degradation. Also, chitosan is easily to be established with other materials and form nerve composite conduits. Thus, this review article tries to compare some new chitosan composite conduits according to the categories of chitosan-materials composite, chitosan-drug-drug delivery system composite and chitosan-nanometal particle composite.

\section{Chitosan composites nerve conduits}

\subsection{Chitosan-materials composite}

\subsubsection{Laminin-chitosan-PLGA conduit[14]}

Ten nanofiber filaments made from laminin were built in the chitosan composite conduit with $2 \mathrm{~cm}$ length, 0.2 $\mathrm{mm}$ conduit wall thickness. Raw chitosan-PLGA was built by $3.5 \%$ chitosan solution. Then the chitosanPLGA tubes were soaked in both polyethyleneimine (PEI) and laminin (LN) solutions several times to form a multilayer PEL/LN film on the conduit's surface. Relative few vascular membranes formed around conduit and no adhesion between conduit and muscle at eight weeks post the surgery. However, at week 12, PEI/LNchitosan-PLGA were found thinner, and there was capillaries formation around the tube.

Also, the interesting thing is no scars formation and no inflammatory response. This experiment group uses EMG to evaluate the degree of nerve regeneration (amplitude means the number of nerve Fibers) because injured nerve fiber cannot transmit the electrical impulse. Only nerve conduit group (NULL) showed $0.8 \mathrm{mV}$ peak amplitude and $1.2 \mathrm{~ms}$ latency, which is less efficacy than the autograft group. However, co-culture of neural stem cells and Schwann cells with PEI/LN-chitosan-PLGA conduit showed better results (amplitude: $1.9 \mathrm{mV}$ and latency: $0.9 \mathrm{~ms}$ at 12 th week). This result may be able to show PEI/LN-chitosan-PLGA is an excellent platform to help Schwann Cell and nerve stem cells function. Compare autograft with PEI/LN-chitosan-PLGA alone. The latter may not be a replacement for autograft according to their efficacy. Nevertheless, chitosan composite tubes are easier to get and also will not cause scars formation and inflammatory response as long as good mechanical properties, which make them a right choice for nerve regeneration when combined with Schwann Cell and nerve stem cell. 
Table 1: The comparison of different conduit materials

\begin{tabular}{|c|c|c|c|c|}
\hline Conduit material & $\begin{array}{l}\text { Material } \\
\text { category }\end{array}$ & Structure & Advantages & Reference \\
\hline Collagen type I & Biological & $\begin{array}{l}\text { Semipermeable collagen wrap } \\
\text { (NeuroMend }\end{array}$ & $\begin{array}{l}\text { Self-curling. They can match the } \\
\text { dimension of the injury site. }\end{array}$ & $2,15,16$ \\
\hline Collagen type I & Biological & $\begin{array}{l}\text { Semipermeable tubular structure. } \\
\left.\text { (NeuroMatrix }{ }^{\mathrm{TM}}\right)\end{array}$ & $\begin{array}{l}\text { Straight gap location is less than } \\
3 \mathrm{~cm} .4-8 \text { months }\end{array}$ & $2,15,16$ \\
\hline Keratin & Biological & $\begin{array}{l}\text { Keratin hydrogels with interconnected } \\
\text { pores (pore sizes ranged from } 20 \text { to } \\
50 \mathrm{~mm} \text { ) }\end{array}$ & $\begin{array}{l}\text { Good for cellular infiltration and } \\
\text { proliferation (Schwann cell) }\end{array}$ & 17 \\
\hline PLA & Synthetic & $\begin{array}{l}\text { Made of a flat but thick sheet with } \\
\text { porosity. }\end{array}$ & $\begin{array}{c}\text { Biodegradable, provide a better } \\
\text { overall organization. }\end{array}$ & 1 \\
\hline PGA-collagen & composite & $\begin{array}{l}\text { Cylindrically woven PGA mesh. } \\
\text { Amorphous collagen layers were } \\
\text { coated on both sides. }\end{array}$ & $\begin{array}{c}\text { Biodegradable, promote cell growth } \\
\text { and proliferation. }\end{array}$ & 18 \\
\hline Gelatin-genipin & composite & $\begin{array}{l}\text { concentric and round tube with a } \\
\text { rough outer surface whereas smooth } \\
\text { inner lumen. }\end{array}$ & $\begin{array}{l}\text { Stable and can be used for gap } \\
\text { longer than } 10 \mathrm{~mm} \text { in the rat. }\end{array}$ & 11 \\
\hline
\end{tabular}

\subsubsection{Chitosan/ hyaluronic acid (HA)[10]}

Chitosan/ hyaluronic acid (HA) has been demonstrated that this composite conduit has the ability to decrease scar formation through the process of nerve regeneration. Chitosan/ hyaluronic acid (CHIT/HA) tube was established in this way:

Using chitosan tube to cover both injury sides of mice sciatic nerve anchored by $6-0$ sutures. $0.2 \mathrm{ml} \mathrm{HA}$ gel injected evenly onto this site both on nerve and conduit. Then the experiment conducts SFI calculation, scar adhesion analysis, electrophysiology evaluation, and histology test. According to SFI result, when compared with the control group, CHIT/HA composite tube showed a significant difference appeared at eight weeks, the SFI value showed a upword trend from week 4 to week 12, which can demonstrate that the nerve function in surgery site improved through the test. At week 12, the test group chitosan/HA showed significant difference with control group. Scar adhesion test analyzed the force size to separate each tissue. According to histology test, CHIT/HA group did appear significant difference to the control group in both eight weeks and twelve weeks. However, CHIT group and HA group failed to show statistical difference than the control group. Thus, it may difficult to demonstrate that chitosan and hyaluronic acid can reduce scar formation separately. In the histology test, to the number of myelinated nerve fibers CHIT/HA group have the most quantity of myelinated axons, and showed significant difference with other groups; to the diameter of mtelinated axons, the test group did show larger diameter of axons in recovery site; to the thickness of myelin sheath, through the figure in the origin article both chitosan and chitosan/HA group showed significant difference with control group. The difference between chitosan/Ha group and chitosan group need to be further analysed.

\subsubsection{Chitosan/ hyaluronic acid (HA)[10]}

Chitin is $\mathrm{N}$-acetylated derivative product of chitosan, which has shorter biodegradation time and more stability.
Carboxymethyl (CM)-chitosan is also a water-soluble, nontoxic, and biodegradable chitosan derivate product. These composite conduits with $12 \mathrm{~mm}$ length, 1.7 inner diameters and $0.4 \mathrm{~mm}$ thickness. They had $82.96 \%$ porosity. Their tensile strength was $0.21 \pm 0.04 \mathrm{MPa}$ and resistance strength to suture was $0.50 \pm 0.04 \mathrm{~N}$. According to Electrophysiological index, single chitin group showed significant difference with composite group and autologous nerve, especially in 20 weeks, composite group showed similar index with golden standard group, which means the artificial nerve graft is more effective in promoting nerve regeneration than chitin alone. Through statistical analysis, composite conduits meet the recovery rate of autograft. While composite conduit have larger resource than autograft and it will not damage other body part. In the future, detail data to demonstrate that it can substitute the use of sutograft should be provided.

\subsection{Chitosan-nanometal particles}

Place the figure as close as possible after the point where it is first referenced in the text. If there is a large number of figures and tables it might be necessary to place some before their text citation. If a figure or table is too large to fit into one column, it can be centred across both columns at the top or the bottom of the page.

\subsubsection{Chitosan-Zinc Oxide Nanocomposite Conduit[19]}

$2 \%$ chitosan solution was used to build a conduit with 2 $\mathrm{mm}$ external diameter, $0.2 \mathrm{~mm}$ wall thickness and $1.4 \mathrm{~cm}$ length. The zinc oxide nanoparticles (average size was $30 \mathrm{~nm}$ ) were coated by using a sputter coated with a gold layer. The sciatic function index (SFI) analyses values in sham-surgery group (SHAM), transected control group (TC), chitosan (CHIT) and chitosan-zinc oxide (CZON) group at each 4,8 and 12 weeks evidenced that the recovery of nerve function was significantly better in CZON than in Chitosan. The results that CHIT group recovered from -90 to -60 , compare with the CZON 
group from -60 to -40 , demonstrated the better recovery rate at 4,8,12weeks. The muscle's weight around nerve tissue was also measured in each group. Chitosan-zinc oxide nanoparticle group showed the great result which is even similar to the SHAM group. However, this article did not compare the difference between CZON and SHAM group.

According to histology data, CZON group revealed the increasing number of Axon fibers and axon diameter $(23703 \pm 2137$ of axon counts fb and $6.41 \pm 0.17$ of axon diameter), while the thickness of myelin sheath remains unchanged with TC and CHIT group.

\subsection{Chitosan-drug composite}

\subsubsection{PCL/Chitosan/Verapamil[20]}

Verapamil is a calcium channel blocker, and it may be able to reduce dysfunction on nerve injury site. The conduit is a chitosan-based composite nanofiber conduit. $\mathrm{PCL} /$ chitosan weight ratios varying from $40 / 60$ to $80 / 20$ are fibered on the mats by electrospinning. PCL have proper viscosity and can be dissolved in a wide range of solutions, so it makes chitosan fibered easier on a sheet. CHIT/Verapamil group is treated $10 \mu 1$ verapamil (100ng/mL). As the result of SFI test and muscle weight measurement,. The SFI of CHIT/verapamil changed from -20 to -12 and $1.3 \mathrm{~g}$ of gastrocnemius muscle, which showed that chitosan group and chitosan with verapamil group did accelerate the regeneration rate of nerve tissue with less nerve function loss and less muscle loss. Also, chitosan and verapamil group showed significant difference with chitosan group and control group in the histology test, CHIT/verapamil group had more axon fibers $(25104 \pm 2287)$ and wider axon diameter (7.78 \pm 0.13$)$. The axons counts fb of control group is $4080 \pm 2105$ and the axon diameter is $3.37 \pm 0.12$, and The axons counts fb of chitosan group is $20307 \pm 2112$ and the axon diameter is $6.19 \pm 0.14$. As a composite containing chitosan and drug, the result showed the drug's function of enhancing nerve regeneration.

\subsubsection{Chitosan/simvastatin/Pluronic hydrogel.[21]}

$F-127$

Chitosan/simvastatin/Pluronic F-127 hydrogel is a kind of chitosan-drug-drug delivery system composite conduit, which make the conduit can help cell adhesion and proliferation by biological chitosan and upregulate the expression of a neurotrophic factor in the friendly and controllable way by the drug-drug delivery system at the same time. Simvastatin can recruit bone marrow stromal cells to injured nerve site and upregulate the expression of neurotrophic factor to help nerve functional recovery. Pluronic F-127 is a new drug delivery vehicle which is biocompatible, low-toxic, and will not stimulate an immune response. Pluronic F-127 hydrogels contains 25 and $50 \mathrm{mg} / \mathrm{mL}$ drug. Chitosan tube was filled with this drug-drug delivery system. The same experimental tests were conducted such as SFI analysis, electrophysiology, gastrocnemius wet weight ratio calculation and detail data about axons. SFI evaluated hollow chitosan conduit and conduit with different concentration drug. $1 \mathrm{mg}$ simvastatin group showed the best functional recovery ability. The $\mathrm{P}$ value is less than 0.005 while greater than 0.001 when compare SIM group with hollow chitosan conduit group. In a certain range, the recovery ability will improve with the increasing of drug amount. According to electrophysiology, $\mathrm{C}+\mathrm{sim}-1 \mathrm{mg}$ also showed the best result, with 10 peak amplitudes of CMAPs. Muscle weight data showed a significant difference between the drug group and the non-drug group. When compare the axonal diameter of myelinated fibers and thickness of myelin sheaths by five groups, the result shoed some similiarity with SFI test. $\mathrm{C}+\operatorname{sim} 1 \mathrm{mg}$ had the best result and in a certain range the recovery ability will go with the concentration of drug.

\section{Discussion}

All chitosan composite conduits demonstrated that they could help nerve regeneration. The main categories of chitosan composite conduits can be divided into chitosan with other biocompatible materials, chitosan with the drug-drug delivery system, and chitosan with nanoparticle metal. Other kinds of conduit materials can give additional properties based on chitosan, such as better mechanical properties, better surface properties, scar formation prevention or even electroconductivity. Drug delivery system may either help tissue regeneration by upregulating the expression of neurotrophic factor by surrounding tissue or prevent fibroblast aggregation and scar tissue formation. Metal nanoparticles give a higher surface area-volume ratio which can increase the electroconductivity. As the sensory transduction on the nerve is an electronic form. Thus, metal added in the conduit also can help tissue regeneration.

This review article compared different chitosan and chitin composite materials. Same experiment test choices make this comparison available, while different experiment must conduct in a different place, time, humidity, and temperature, the man who operates the surgery also different. This results in great difficulty to make this comparison. This article set hollow chitosan group as the standard group to compare how better the chitosan composite changed such as more significant axonal numbers, a wider diameter of myelinated nerve fibers et.al. This gives a brief comparison between all three categories' chitosan composite although not that precise and convincing. Take all kinds of chitosan composite conduits and compare them together in the same experiment by the same person is necessary for the future.

Chitosan composite conduits can be compared in physical characteristics such as the conduit structure and the established methods. The length of the conduit often based on the length of the injury site. Actually, most research uses $12 \mathrm{~mm}$ conduit to bridge $10 \mathrm{~mm}$ nerve gap of rats. Both sides have $1 \mathrm{~mm}$ for nerve suture onto the conduit. $10 \mathrm{~mm}$ is a critical gap for rats which means if the gap longer than $10 \mathrm{~mm}$, it will be very hard or even 
impossible to regenerate. $10 \mathrm{~mm}$ is equal to $10 \mathrm{~cm}$ of human's critical nerve gap. The wall thickness ranges from $0.2 \mathrm{~mm}$ to $0.4 \mathrm{~mm}$ with $2 \mathrm{~mm}$ in external diameter. Most chitosan composite conduit tried to increase the porosity of the conduit, just like bone tissue scaffold, which can help nutrient transport readily. To construction method, chitosan-material often established in a mixed solution. $2 \%(\mathrm{w} / \mathrm{v})$ while Glacial acetic acid often adds into this chitosan mixed solution to increase the water-solubility of $3.5 \%(\mathrm{w} / \mathrm{v})$ chitosan. Synthetic materials like PLGA or biological materials like chitin or even zinc oxide nanoparticles are added into the chitosan/acetic acid solution first following by stirring and injecting into a mold, then freeze-drying in the machine. Hyaluronic acid (HA) gel is considered as a kind of extracellular matrix, so it will be injected onto the surface of chitosan conduits after inserting into the injury site. Verapamil, the same as HA gel, both of them only have the function on nerve regrowth, while having no function on conduit establishment. Thus, they just added into the chitosan conduit after the surgery. Chitosan/simvastatin/Pluronic F-127 hydrogel is quite different because of the existence of the drug delivery system, which is required to be added into the solution before conduit establishment. Chitosan is nonelectroconductivity, which give the difficulties to research the whole structure of the composite conduits under the scanning electron microscope (SEM). Fluorogold can be coated onto the surface of the conduit and give the response to the electron signal, which can help us get perfect images.

SFI test and electrophysiology are also relevant data need to be compared. Sciatic functional index (SFI) is prevalent and convective data to assess the functional nerve recovery. Setting the chitosan group in 12 weeks as the standard group, Laminin-chitosan-PLGA conduit group did not show the data of SFI, Chitosan-Zinc Oxide Nanocomposite conduit showed CZON group recover $162.5 \%$ faster than CHIT group. PCL/Chitosan/Verapamil showed $112.5 \%$ better than CHIT group. Chitosan/ hyaluronic acid (HA) 105\% faster than CHIT group. Chitosan/simvastatin/Pluronic F-127 hydrogel showed $218.8 \%$ faster than CHIT group. To the electrophysiology, only the nerve can conduct the electrical signal so that the electrophysiology can demonstrate the recovery rate of the nerve. Only two conduits did the electrophysiology test, and PEI/LNchitosan-PLGA conduit showed better results: amplitude: $1.9 \mathrm{mV}$ and latency: $0.9 \mathrm{~ms}$ at 12th week. As the comparison result, Chitosan/simvastatin/Pluronic F-127 hydrogel, the chitosan-drug delivery system may have better results and compare PEI/LN-chitosan-PLGA with chitin-CM-chitosan, the former one may be better.

To the histology test, as L. J. Chamberlain et al. [22] reported that the maturity of regenerated nerves were often represented by average density and average diameter of regenerated myelinated nerve fibers. The evaluation will be quite validated in these dataAll the composite conduits groups showed significant difference with the control group. When compared with chitosan group, c+sim $1 \mathrm{mg}$ group had $168.0 \%$ larger axonal diameter of myelinated fibers and $200.0 \%$ thickness of myelin sheaths. Chitosan/HA group showed similar results in the histology test. Chitosan/verapamil had $123.6 \%$ higher axonal number, $125.7 \%$ larger axonal diameter of myelinated fibers and $124 \%$ thickness of myelin sheaths. CZON had $112.8 \%$ higher axonal number and $172.7 \%$ larger axonal diameter of myelinated fibers, while to the thickness of myelin sheaths both CHIT and CZON had the same data.

All the test showed that chitosan composite conduits have a better result in nerve regeneration comparing with chitosan group. Just according to the data comparison above, Chitosan/simvastatin/Pluronic F-127 hydrogel appeared the best result of the nerve regrowth. However, as I said before, this just an unprofessional comparison, many changes like temperature, mice difference, humidity et al. made the result quite different.

\section{Conclusion and future perspective}

A new study not only combines a few kinds of materials to give the composite conduit just physical and chemical properties improvements such as mechanical properties, hydrophily, and charge. Most composite conduits are trying to reduce the formation of scar, preventing of fibroblast and inflammation and supplement of neuron growth factor to the injury site. In the future, composite conduit combine with cell culture method and drug-drug delivery system may be the major solution to nerve defect.

\section{References}

1. Kim, S. M. et al. Biodegradable Nerve Guidance Conduit with Microporous and Micropatterned Poly ( lactic- co -glycolic acid ) -Accelerated Sciatic Nerve Regeneration. 1800290, 1-14 (2018).

2. Dursun, T., Yucel, D. \& Hasirci, N. Peripheral nerve conduits : technology update. 405-424 (2014).

3. Oliveira, C. et al. Evaluation of biodegradable polymer conduits - poly ( L -lactic acid ) - for guiding sciatic nerve regeneration in mice. Methods 99, 28-36 (2016).

4. Hsueh, Y. et al. Biomaterials Functional recoveries of sciatic nerve regeneration by combining chitosancoated conduit and neurosphere cells induced from adipose- derived stem cells. Biomaterials 1-11 (2013). doi:10.1016/j.biomaterials.2013.11.081

5. Godard C. W. de ruiter, M.d., 1 Martijn j. a. Malessy, M.d., Ph.d., 1 MiChael j. yaszeMski, M.d., Ph.d., 2 anthony j. Windebank, M.d., 3 and robert j. sPinner, M. d. Designing ideal conduits for peripheral nerve repair. 26, 1-9 (2009).

6. Muheremu, A. \& Ao, Q. Past, Present, and Future of Nerve Conduits in the Treatment of Peripheral Nerve Injury. 2015, (2015).

7. Ronchi, G. et al. Chitosan Tubes Enriched with Fresh Skeletal Muscle Fibers for Primary Nerve Repair. 2018, (2018).

8. Jiang, Z. et al. Rat sciatic nerve regeneration across 
a $10-\mathrm{mm}$ defect bridged by a chitin/CM-chitosan artificial nerve graft. Int. J. Biol. Macromol. \#pagerange\# doi:10.1016/j.ijbiomac.2019.02.080

9. Bamba, R. et al. A Novel Conduit-Based Coaptation Device for Primary Nerve Repair. 7454, (2017).

10. Li, R. et al. Chitosan conduit combined with hyaluronic acid prevent sciatic nerve scar in a rat model of peripheral nerve crush injury. 4360-4368 (2018). doi:10.3892/mmr.2018.8388

11. Chen, Y. et al. An in vivo evaluation of a biodegradable genipin-cross-linked gelatin peripheral nerve guide conduit material. 26, 39113918 (2005).

12. Liu, B. Fabrication and evaluation of a biodegradable proanthocyanidin-crosslinked gelatin conduit in peripheral nerve repair. (2008). doi:10.1002/jbm.a.31916

13. Golafshan, N., Kharaziha, M. \& Fathi, M. Tough and conductive hybrid graphene-PVA: Alginate fi brous scaffolds for engineering neural construct. Carbon N. Y. 111, 752-763 (2017).

14. Li, Y. U., Yu, Z., Men, Y., Chen, X. \& Wang, B. Laminin - chitosan - PLGA conduit co - transplanted with Schwann and neural stem cells to repair the injured recurrent laryngeal nerve. 1250-1258 (2018). doi:10.3892/etm.2018.6343

15. Peripheral, S. Nerve repair.

16. Siemers, F. \& Maila, P. The Clinical Use of Artificial Nerve Conduits for Digital Nerve Repair : A Prospective Cohort Study and Literature Review. 1, 55-61 (2009).

17. Sierpinski, P. et al. The use of keratin biomaterials derived from human hair for the promotion of rapid regeneration of peripheral nerves. 29, 118-128 (2008).

18. D, K. M. M. et al. Peripheral nerve regeneration across an $80-\mathrm{mm}$ gap bridged by a polyglycolic acid ( PGA )- collagen tube filled with laminin-coated collagen fibers: a histological and electrophysiological evaluation of regenerated nerves. 868, 315-328 (2000).

19. Article, O. Effects of Chitosan-Zinc Oxide Nanocomposite Conduit on Transected Sciatic Nerve: An Animal Model Study. 5, 240-248 (2017).

20. Article, O. Effect of Local Administration of Verapamil Combined with Chitosan Based Hybrid Nanofiber Conduit on Transected Sciatic Nerve in Rat. 7, 28-34 (2019).

21. Guo, Q. et al. Chitosan conduits filled with simvastatin / Pluronic F-127 hydrogel promote peripheral nerve regeneration in rats. 1-13 (2017). doi:10.1002/jbm.b.33890

22. Chamberlain, L. J., Yannas, I. V, Hsu, H., Strichartz, G. \& Spector, M. Collagen-GAG Substrate Enhances the Quality of Nerve Regeneration through Collagen Tubes up to Level of Autograft. 329, 315-329 (1998). 